\title{
Colliding Events: Art Projects at the Large Hadron Collider
}

\author{
Chris Henschke \\ Monash University \\ Faculty of Art Design \& Architecture \\ 900 Dandenong Road, Caulfield East \\ VIC 3145 \\ Australia \\ christopher.henschke@monash.edu
}

\begin{abstract}
In this paper the author describe two media art projects developed in 2014 during his "art@CMS" residency at the European Organization for Nuclear Research Compact Muon Solenoid experiment, as part of his PhD by research project, titled 'The Universe is my Laboratory'.
\end{abstract}

Media art. Particle physics. Scientific and creative visualisation

\section{DETECTORS AND DATA}

The Nature of the Apparatus is an audio-visual artwork, five minutes in duration, produced by Chris Henschke during his "art@CMS" residency at the European Organization for Nuclear Research (CERN) in 2014. The work conveys both experimental and experiential aspects of the Large Hadron Collider (LHC). The LHC is the largest scientific experiment in the world, and probes the fundamental nature of the material universe, through the focusing of trillions of electron volts of energy on unimaginably small subatomic particles that are accelerated to almost the speed of light around the twenty seven kilometre accelerator ring. When the particles collide with each other, immense energies are released for an instant of time, which are captured by gargantuan instruments such as the Compact Muon Solenoid (CMS) detector. This was one of the devices that proved the existence of the Higgs Boson, also known as the "God Particle".

The artwork is derived from ultra-high definition video shot by Henschke and CMS physicist $\mathrm{Dr}$ Michael Hoch at various locations across CERN, including areas that are usually inaccessible. The locations ranged from old near-abandoned experimental zones, such as a hundred deep metre shaft what the CERN scientists refer to as "The Black Hole", to contemporary experiments, including the 15,000 ton CMS detector situated 100 metres underground. As well as conveying the almost overwhelming scale and complexity of the science and technology involved, the video also expresses the unimaginable energies produced in the giant apparatus. However, the material that was captured on video is largely of inert devices, as it is not possible for anyone to be on site when the LHC is active, as the radiation levels emanating from the particle beam and collisions would be fatal.

The footage is manipulated in a way that uses the "sound" of the LHC beam to control the flow of the video (see Figure 1).

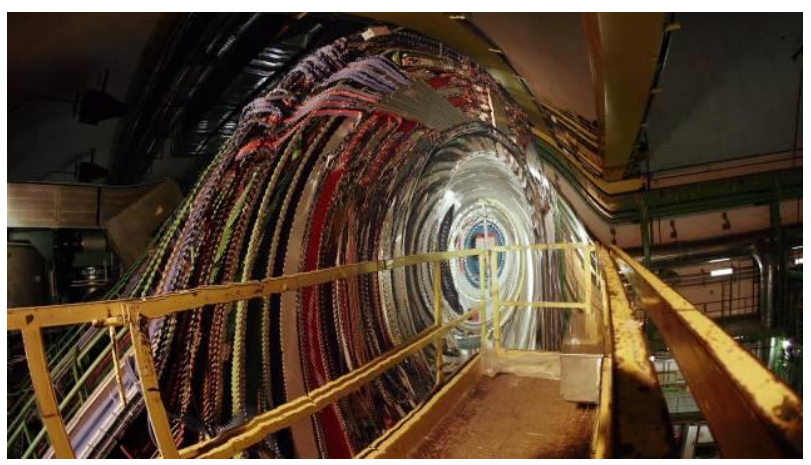

Figure 1: Still from The Nature of the Apparatus

This sound is actually the electromagnetic wave that is vibrating transversely along the energy beam in the LHC, analogous to the way a vibration passes along a violin string. In a unique experiment, collaborating accelerator physicist Ralph Steinhagen 'plucked' the LHC's beam and recorded the vibrations it made. Interestingly, the frequency of this transverse wave vibration is within the audible range of human perception, so the sound of the LHC can be directly heard, in the same way that an electrical audio signal can be 
heard when it is plugged into to a speaker. This sound / energy vibration is used to affect the video in a way that algorithmically spatializes the sound dynamics by mapping (video) time across (screen) space. Using Adobe After Effects software, the amplitude of the sound wave is analyzed and turned into numerical data. An expression script, based on the Java programming language, translates the amplitude data into timedisplacement data. This manipulates the source video footage in a way that maps horizontal or vertical slices of the footage onto the screen, with the resultant effect that the greater the sound intensity is, the larger the temporal splicing. Thus, depending on the movement of the source footage, the resultant video spatiotemporally compresses or extends the footage and thus visually compresses or extends the space in the video footage.

\subsection{PHENOMENA and knowledge}

By embedding the energy within the footage of the apparatus that produces it, they become conceptually connected into a state which the quantum physicist Niels Bohr calls "phenomena". This manifests Bohr's philosophical standpoint on the relationship between the scientific devices and the objects of their investigation - for Bohr, the interaction between object and apparatus 'forms an inseparable part of the phenomenon' (Barad 2007, p. 119). As physicist and philosopher Karen Barad states, 'apparatuses are not passive observing instruments, on the contrary, they are productive (and part of) phenomena', yet Bohr does not define 'what precisely constitutes the limits of the apparatus' (Barad 2007, p. 142). Is it the detector, the computer terminal, the display screen, the scientist viewing it, the organizations responsible for funding the project? When dealing with the boundary between the quantum and macroscopic worlds, the nature of the apparatus becomes an entanglement of matter and meaning. Bohr states that we are 'constrained by our inability to construct experimental apparatus in anything other than classical dimensions, [thus] we are denied an insight into the 'true' quantum world. What we get instead is the quantum world as reflected in the mirrors of our classical apparatus' (Pais 1991, p. 97). Unlike the classical or macroscopic world, where we can ask definite questions about what things are, in the quantum realm, one should ask:

\footnotetext{
Does the electron (or any other object) behave like a particle or like a wave? That question is answerable, but only if one specifies the experimental arrangement by means of which 'one looks' at the electron. That is what Bohr meant ... when he said; 'An independent reality in the ordinary [that is, classical] physical sense can ... neither be ascribed to the phenomena nor to the agencies of observation' (Pais 1991, p. 314).
}

Barad's response to this fundamental issue comes from a post-humanist philosophical standpoint, in that 'apparatuses are boundary making practices', and that ultimately we are not separate entities: 'posthumanism doesn't presume the separateness of any-'thing', let alone the alleged spatial, ontological, and epistemological distinction that sets humans apart'. Our "agency" or decisions and actions, are 'intra-active' dynamic reconfigurings of the topology of spacetimematter relations (Barad 2007, pp. 136-140). The universe and us are not separate, we are in a continual dance of defining each other. As the scientist and philosopher Michael Polanyi states, 'The knower does not stand apart from the universe, but participates personally within it' (Rheinberger 1997, p. 23).

The contemporary experimental apparatuses such as the 'megalithic machines' at CERN, where 'each detector is a world in itself' (Galison 1997, p. 688), allow us to interact with the very fundamentals and extremes of our universe. The spatial, temporal and energetic dynamics that occur in the core of CMS can be seen as a form of the sublime. This is a sensation that is described as the apprehension that which is beyond humanity's grasp or limits of understanding. The philosopher Immanuel Kant posits two types of sublime: the "mathematical" which deals with scale, and the "dynamic" which deals with the might and power of nature. According to Kant, we find ourselves at the limits of our ability to intuitively grasp or aesthetically measure such states of the sublime due to the 'limitations of the finite, human faculties of sensibility' (Kant 1790). The LHC encapsulates both forms of the sublime, by its unimaginable complexity and the tremendous energy it creates. However, this is a kind of inversion of the Kantian sublime of scale and dynamics - it is the sublime of the near-infinitely small yet near-infinitely energetic.

\subsection{Epistemic Objects}

The Large Hadron Collider physically manifests another limit, the limit of knowledge itself, as the experiments undertaken there are literally at the boundaries of knowledge and the scientific understanding of the universe. The LHC brings together and tests the theoretical and experimental aspects of knowledge, manifested through the combination of the devices used and the outcomes of the experiments. From this point of view, it can be seen that, as Barad states, 'theorizing, like experimenting, is a material practice' (Barad, p. 55). The LHC itself can be described as an object of knowledge, or 'epistemic object', a term used by scientist Hans Rheinberger to describe 'any scientific objects of investigation that are at the centre of a research process and in the process of being materially defined. Objects of knowledge are characteristically open, question-generating and 
complex. They are processes and projections rather than definitive things' (Knorr Cetina 2001, p. 191).

Such a state is succinctly illustrated with a quote from Polanyi: 'This capacity of a thing to reveal itself in unexpected ways in the future, I attribute to the fact that the thing observed is an aspect of reality, possessing a significance that is not exhausted by our conception of any single aspect of it. To trust that a thing we know is real is, in this sense, to feel that it has the independence and power for manifesting itself in yet un-thought of ways in the future.' (Polanyi 1966, pp. 32-33)

Expanding on this description, Rheinberger (1997) states the following:

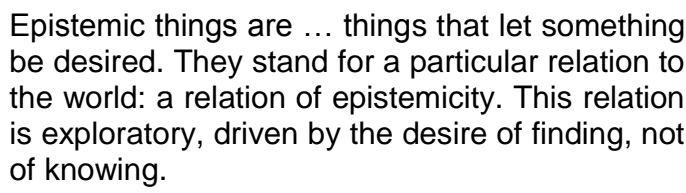
be desired. They stand for a particular relation to the world: a relation of epistemicity. This relation is exploratory, driven by the desire of finding, not of knowing.

Experimenters are specialists in arranging situations in which finding becomes possible. Scientific finding neither obeys the logic of chance nor that of necessity. It obeys a logic of its own, composed of elements of both, and in so doing, undoes the stochastic rigor of the one and the deterministic rigor of the other. It is a game of eventuation, an engagement with the material world that, on the one hand, requires intimacy with the matter at hand, and, on the other, disentanglement, the capacity of rendering strange-of estrangement. I am convinced that the poet's and the artist's activities share the basic feature of this epistemic condition.

I stress the agency of the material one engages with. In this respect, what unites research materials is eventuation: through a material we step away from what is there, sidestep what is actually realized, toward the unrealized by taking advantage of, to go back to the telling phrase Polanyi uses, its "independence and power for manifesting itself in yet un-thought of ways in the future." It is the conviction that the sciences and the arts nourish themselves from the materials they engage with, constantly stepping away from and over what is already there, without ever being able to precisely anticipate the path they are not merely taking, but creating as they tread.

Bringing together the audio and visual material in The Nature of the Apparatus can be seen as a creative manifestation of an epistemic object, or even as a 'readymade', in the sense of the term coined by the French mathematician Poincare, the 'grandfather of relativity' (Perelló \& Altaió 2008, p. 233). Poincare used the term 'tout fait objects [readymades] to describe 'the epiphanies resulting from a barrage of pre-established ideas' (Perelló \& Altaió 2008, p. 235). According to Poincaré's formulation of the nature of human creativity, following an intensive but more or less random input of study, ideas appear to sort themselves out in what he calls the unconscious mind. There follows "tout fait," the illuminating flash of insight.

From the new materialist perspective of philosopher Manuel DeLanda, light and sound are forms of illumination and expression applicable not only to us. DeLanda states: 'The characteristics [of wavelength components and vibration frequency] allow both light and sound to produce distinctive effects on animal and human brains, effects that may be used for expressive purposes... by human artists. But possession of a nervous system is not necessary to make expressive use of colour or sound. Even humble atoms can interact with light... in a way that literally expresses their identity' (DeLanda 2011, p. 7).

\section{OBSERVATION AND EXPRESSION}

Edge of the Observable is an audio-visual artwork which explores the limits of materiality and knowledge, through an experimental remanifestation of data taken from experiments at the CMS detector (see Figure 2). The work seeks to manifest such sublime and dynamic expressive qualities that are present within the LHC through the extraction of dynamic and expressive qualities from data captured by the CMS experiment. It also plays with the concept of the 'golden event', a term used in particle physics to describe a perfectly captured image of a rare or important phenomenon. However, this type of visual knowledge has been largely superseded by the types of numeric and statistical analysis used in modern particle physics. Physicist Peter Galison describes the tension between these 'image and logic traditions' (Galison 1997, pp. 19-25):

Images [captured in bubble chambers] are presented, and defended, as mimetic - they purport to preserve the forms of things as they occur in the world. ... Against this mimetic tradition...[is].. the "logic tradition" which has used electronic counters coupled in electronic logic circuits... to make statistical arguments for the existence of a particle or effect. The clash of image and logic traditions [is the clash of the] golden event versus statistical demonstration, the objectivity of passive registration versus the persuasiveness of experimental control, vision versus numbers, and photography versus electronics. 

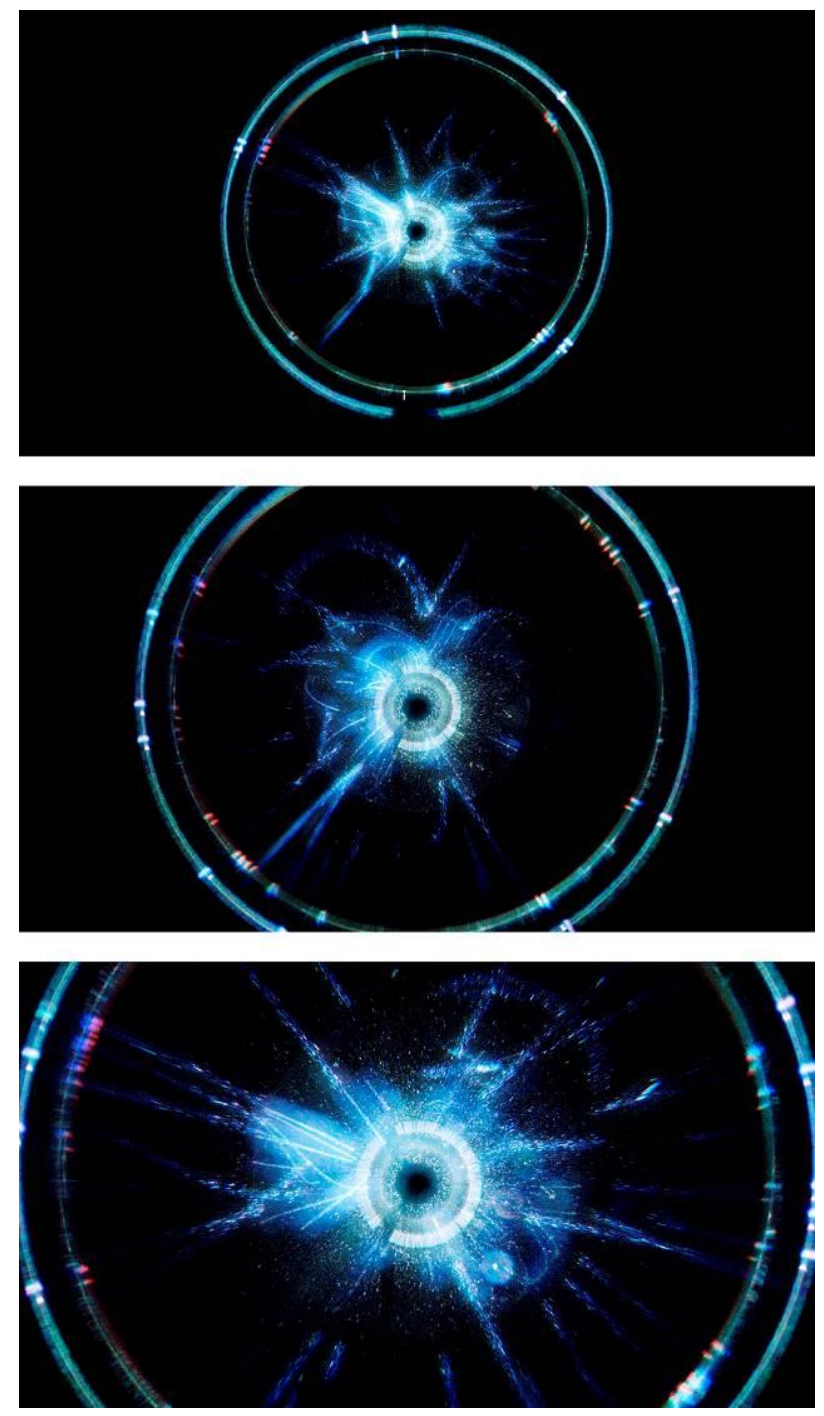

Figure 2: Still sequence from Edge of the Observable

Heralding perhaps a renaissance in scientific imaging, experiments such as CMS combine image and logic based analysis, in a 'shift from the pure to the hybrid' experiment and from 'the modern to the postmodern laboratory' (Galison 1997, p. 553). The "Time Projection Chamber", which is the heart of the CMS detector, works in a way that is poetically described by Galison that creates 'an image falling through space' (Galison 1997, p. 553). Time projection chambers themselves are 'high-tech bricolages' (Galison 1997, p. 550) of both bubble chamber and electronic logic devices. These these hybrid detectors contain the 'historical epistemology' of experimental particle physics, where golden events are 'produced by electronics, fished by a computational net out of the ocean of microphysical debris' (Galison 1997, p. 551).

Edge of the Observable specifically portrays one of the billions of particle collisions that occur daily in the CMS detector: event 416497095 of event run 46944. This event in itself has no particular scientific value, but it has compelling expressive qualities. The data taken from this collision event is the source material for the artwork; however it is manifested through an experimental physical setup. Taking the basic form of a physics experiment, the data is emitted as light from an energy source; it is then modulated through an optical lens-like device; and is then captured and recorded by a detection device. By finely tuning the physical variables of the experiment, plus some minor digital postproduction adjustments, the resultant output contains an essence of both the setup of the artwork as well as that of the LHC experiments. The accompanying sound is that of the LHC beam tune, which is simply pitch-shifted and equalized to enhance its expressive qualities. The final artwork is recorded in ultra high-definition video and presented as a twelve minute looping audio-visual sequence.

\subsection{DYNAMIC OUTPUTS}

In summation, the work incorporates the ideas, methods, and results of experimental physics, and has science and knowledge embedded within it, but is in itself not science or scientific illustration; rather it is cultivated and nourished from the materials it engages with. The ultimate aim of the work, through critically engaging with the science, is to challenge the physicists' ownership of the forms created in the detectors. The work seeks to capture and distil the formal and dynamic qualities of the particle collisions in a way that speaks to a universal audience, and transcends the ultraspecialist realm of particle physics.

Edge of the Observable was exhibited at the 'art@CMS vernissage', June 26-30, 2014. The event was attended by about eight hundred CERN scientists. With funding from CMS, Chris Henschke and Michael Hoch also exhibited works from the 'art@CMS' residency program, at the International Conference on High Energy Physics (ICHEP14), in Valencia, Spain, from July 1-7, 2014. At the conference, the work was set up in the style of a science information stand, under a banner emblazoned with the words 'Colliding Events', plus scientific style posters and brochures. Displaying the work as a mock science installation allowed dialogues to be initiated with attending scientists, several of whom thought it was some kind of scientific display, and who may not have been so interested if it was explicitly portrayed as an artwork. This has led to ongoing communication with several physicists, and has lead to further projects. The work was also shown at the 'Small Infinite' exhibition at the University of Southampton John Hansard Gallery, from August 5 to 28. The show, curated by Lanfranco Aceti and Vince Dziekan, featured works from the series One Second Drawings by John Latham (1921-2006), a 
British artist who developed a unique oeuvre derived from a personal theory of cosmology. Exploring the theme of the infinitesimally small, the exhibition contained various still and time-based works by a range of contemporary artists.

\section{REFERENCES}

Barad, K. (2007) Meeting the Universe Halfway: Quantum Physics and the Entanglement of Matter and Meaning. Duke University, Durham.

DeLanda, M. (2012) Matter Matters. Domus, Rozzano.

Galison, P. (1997) Image and Logic: A material culture of microphysics. University of Chicago Press, Chicago.

Burnham, D. (n.d.) Kant's Aesthetics. http://www.iep.utm.edu/kantaest/\#SH2c (retrieved 20 October 2014).
Knorr Cetina, K. (2001) Objectual Practice. In Schatzki, T., Knorr Cetina, K., \& von Savigny, E., (eds.), The Practice Turn in Contemporary Theory. Routledge, London.

Pais, A. (1991) Niels Bohr's Times: In physics, philosophy, and polity. Clarendon Press, Oxford.

Perelló, J. \& Altaió V. (2008) Physics of Aesthetics: A Meeting of Science, Art and Thought in Barcelona. Leonardo, 41(3), 233-237.

Polanyi, M. (1966) The Tacit Dimension. Doubleday \& Co., New York.

Rheinberger, H. (n.d.) On Epistemic Things, and Around. http://wdwreview.org/think/on-epistemicobjects-and-around// (retrieved 10 January 2015).

Rheinberger, H. (1997) Towards a History of Epistemic Things: Synthesizing proteins in the test tube. Stanford University Press, Stanford. 